\title{
The Low-Mass Dark Matter Frontier
}

\author{
Traditional searches for dark matter rely on giant detectors that look for \\ particles heavier than a proton, but sights are turning to smaller \\ experiments with sensitivity to lighter-mass particles.
}

\author{
By Rouven Essig
}

$\square$ xtensive evidence suggests that a staggering $85 \%$ of the matter in our Universe is dark matter. This substance interacts gravitationally with itself and with ordinary matter, but whatever other interactions it has with regular matter have eluded detection. For this reason, the presence of dark matter is inferred indirectly-from the properties of the cosmic microwave background, from the distribution and velocities of stars, galaxies, and galaxy clusters, and from the gravitational lensing of distant light sources.

The most plausible explanation for dark matter is that it consists of one or more new particles that are not described by the standard model of particle physics. Yet despite decades of searching, no convincing laboratory evidence for dark matter exists. Many basic properties of dark matter particles therefore remain unknown, such as their mass, spin, and whether they have nongravitational interactions with known standard model particles.

One path forward is to ramp up the sensitivity of existing detectors, which mostly look for dark matter with mass close to or heavier than the proton $\left(1 \mathrm{GeV} / \mathrm{c}^{2}\right)$. Roughly speaking, bigger is better for these experiments because sensitivity goes up with detector size. But another option is to branch out by considering other types of dark matter and new methods to look for them. An appealing possibility-and the focus of this article-is dark matter whose mass lies in a window just below that of the proton. Thanks to progress in particle theory and with new ultrasensitive sensors, a new crop of smaller detectors will look for these lighter dark matter candidates. Already, researchers have had exciting success using these detectors to

\section{ARTICLE TAKEAWAYS}

There is a crucial need to explore dark matter candidates across a much wider mass range than that of the WIMP.

New detection schemes will allow researchers to search for so-called sub-GeV dark matter, which can be up to one million times lighter than the mass of the proton.

The new schemes rely on inelastic collisions between a dark matter particle and electrons or nuclei in various materials, such as semiconductors, scintillators, and superconductors.

probe the new mass range (Fig. 1). An essential next step is to find and implement ways of boosting the detectors' sensitivity.

\section{Casting a Wider Net for Dark Matter}

For several decades, the favorite dark matter candidate has been the weakly interacting massive particle, or WIMP. A WIMP interacts with ordinary particles through the weak force, and its mass is expected to lie somewhere between the proton mass and a mass one thousand times larger. WIMPs are attractive dark matter candidates. They can be produced from collisions of known particles shortly after the big bang in an amount consistent with cosmological and astrophysical observations. Further enthusiasm for WIMPs comes from supersymmetry and other models that aim to "fix" the standard model because they predict WIMPs and other new particles in the WIMP mass range.

Despite their meek name, WIMPs have dominated the experimental search for dark matter. In particular, dozens of experiments have attempted to detect a dark matter particle from our Milky Way halo through its scattering off a nucleus in 


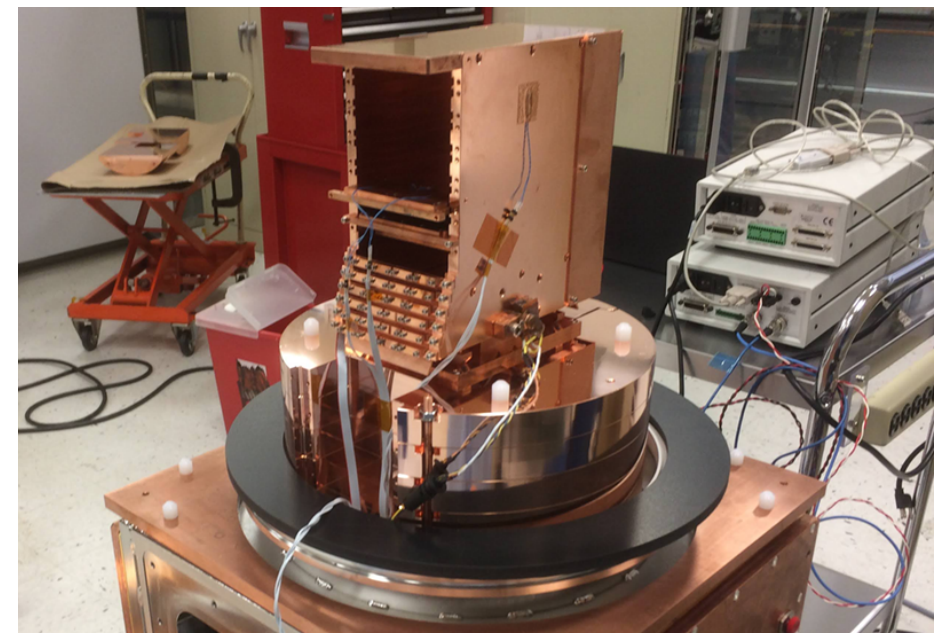

Figure 1: Some dark matter hunters have turned their attention to detecting particles lighter than WIMPs. One such experiment, known as SENSEI, relies on detectors consisting of charge-coupled devices and is shown here under construction at Fermilab.

Credit: SENSEI Collaboration/Fermilab

some target material. These "direct-detection" experiments, some of which will soon consist of multi-ton targets, are typically placed deep underground. In addition, thick shielding helps to avoid harsh cosmic rays and radioactive backgrounds, which can mimic dark matter events.

There is a crucial need, however, to explore dark matter candidates across a much wider mass range than that of the WIMP. For one, no clear sign of dark matter has emerged in many dozens of experimental WIMP searches. Two, none of the particles that might accompany WIMPs, such as

supersymmetric particles, have been discovered at the Large Hadron Collider or elsewhere. And three, many compelling non-WIMP dark matter candidates exist. A possibility that is receiving increased attention is that of "sub-GeV" dark matter, which has a mass between $1 \mathrm{keV} / \mathrm{c}^{2}$ and the mass of the proton. Unlike WIMPs, sub-GeV dark matter candidates interact with regular matter through some new force (rather than the weak force). Theorists have by now discovered a myriad of ways that such dark matter could have been produced with an abundance consistent with astrophysical observations. Moreover, these candidates could easily have evaded detection because of a glaring blind spot in our experimental searches so far [1].
The light mass of sub-GeV dark matter does, however, pose a challenge to detection. Dark matter halo particles move at nonrelativistic speeds-typically about $300 \mathrm{~km} / \mathrm{s}$, or $10^{-3} \mathrm{c}$. A major challenge, therefore, in detecting sub-GeV dark matter from a halo is that it does not have much kinetic energy; for example, a dark matter particle with a mass of $2 \mathrm{MeV} / \mathrm{c}^{2}$, has a kinetic energy of only about $1 \mathrm{eV}$. Moreover, the traditional WIMP search looks for dark matter particles scattering off a nucleus elastically. But much as a flying table-tennis ball transfers only very little energy to a billiard ball, so too would a low-mass dark matter particle transfer very little energy to a recoiling nucleus in an elastic collision ( $\sim 0.4 \mathrm{meV}$ for a 2 $\mathrm{MeV} / \mathrm{c}^{2}$ dark matter particle scattering off a xenon nucleus). Such small energies are impossible to measure with today's sensors.

Fortunately, many other interactions are possible between dark and ordinary matter, which allow for a much larger energy transfer. These interactions often lead to qualitatively new signals-distinct from those generated by an elastic scattering event from a nucleus.

\section{New Signals for Detecting Sub-GeV Dark Matter}

A promising idea for finding these new signals exploits interactions between dark matter and a target that are inelastic. These interactions often allow the transfer of a sizable fraction of the particle's kinetic energy to the target, thus leading to a signal that is, in principle, measurable. Dark matter particles can interact inelastically with an electron in, for example, atoms $[2,3]$, crystals [2, 4-8] molecules [9], superconductors [10], or Dirac materials $[11,12]$. They can also interact with nuclei in atoms $[13,14]$, molecular systems $[2,15]$, crystals $[7,8,13,14$, 16], or superfluid helium [17]. The signals resulting from such interactions depend on the precise target material, the dark matter particle's mass, and the particle's interactions with known matter. These signals can, for example, consist of one or a few electrons, electron-hole pairs, photons, phonons, magnons, or plasmons. For instance, a $2 \mathrm{MeV} / \mathrm{c}^{2}$ dark matter particle hitting an electron in a silicon crystal would typically transfer a significant fraction of its kinetic energy to this electron. The transferred energy would then excite the electron from the valence band to the conduction band, across the $\sim 1$-eV crystal band gap, allowing it to be measured. Materials with energy gaps as low as $1 \mathrm{meV}$ can be excited by dark matter as light as $1 \mathrm{keV} / \mathrm{c}^{2}$. A similar approach could be used to detect 


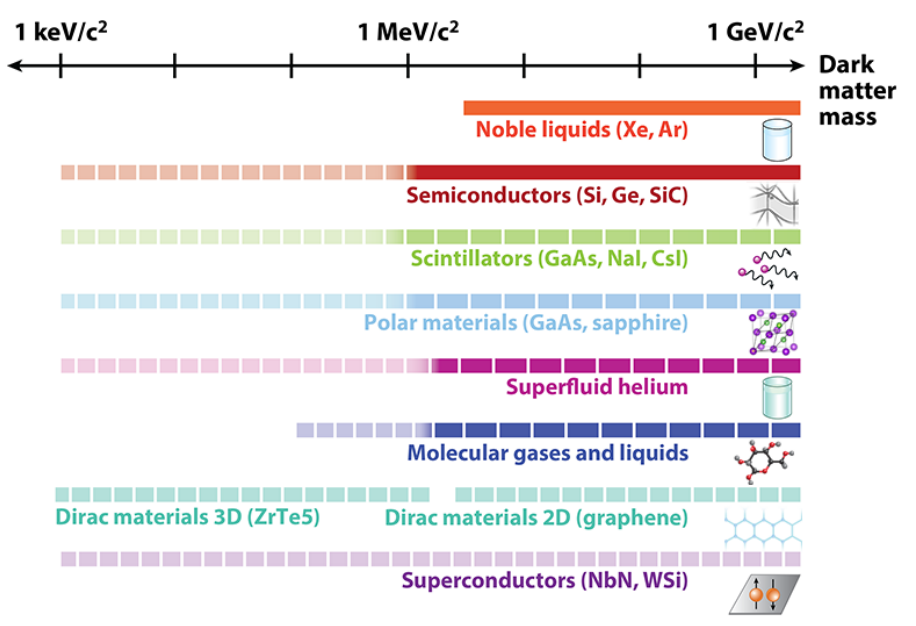

Figure 2: Various materials for detecting sub-GeV dark matter are in use (solid lines) or under consideration for the near term or long term (long-dashed and short-dashed lines, respectively). The horizontal scale indicates the mass-sensitivity range.

Credit: R. Essig/Stony Brook University; APS/Carin Cain

so-called bosonic dark matter, such as a dark photon, an axionlike particle, or a scalar particle, with masses down to 1 meV. In particular, in silicon, a bosonic dark matter particle as light as $1 \mathrm{eV} / \mathrm{c}^{2}$ can be absorbed (instead of scattered), delivering to the electron an energy corresponding to the particle's mass and exciting the electron across the crystal band gap.

Some target materials for detecting sub-GeV dark matter, such as liquid xenon, liquid argon, silicon, and germanium, have been used previously for WIMP searches. Other proposed target materials would be new to the dark matter search community. These include new scintillators, superconductors, superfluid helium, Dirac materials, polar materials, diamond, silicon carbide, aromatic organic targets, and molecular gases (Fig. 2). What's needed, however, is a full description of the signal produced by a dark matter particle interacting with these materials in terms of the particle's properties. In addition, these signals need to be calibrated experimentally. This work, which is in some cases already underway, requires a joint effort between researchers in particle, astroparticle, condensed matter, and atomic-molecular-optical physics.

\section{Promising Detectors}

An effective search for low-mass dark matter requires a detection medium and a sensor with at least three properties. First, the medium has to react to the deposition of tiny amounts of energy. Second, the medium must be scalable to a large number of atomic "targets" to increase the chance of capturing a rare interaction with a dark matter halo particle. Finally, the sensor and medium must have an exquisitely low rate of detector-induced backgrounds ("dark counts"). Physicists have made enormous progress over the past few years in building the first generation of ultrasensitive detectors that are able to search for dark matter with masses as low as $1 \mathrm{MeV} / \mathrm{c}^{2}$, but much remains to be done.

The first demonstration of sensitivity to single electrons came with data from a roughly 10-kg target consisting of liquid xenon, which has an excitation energy of around $12 \mathrm{eV}[3,18]$. Both argon and larger xenon-based detectors have subsequently shown impressive sensitivity to certain dark matter candidates as light as around $5 \mathrm{MeV} / \mathrm{c}^{2}[19,20]$, but further progress is currently hampered by backgrounds, as explained below.

One device that has seen incredible progress recently, includes a special "Skipper" charge-coupled device (CCD) [21, 22], consisting of millions of silicon pixels that are $15 \mathrm{~mm} \times 15 \mathrm{~mm}$ $\times 675 \mathrm{~mm}$ in size. This device is able to precisely measure the number of electrons in each pixel, providing sufficient sensitivity to spot a collision between $\mathrm{MeV} / \mathrm{c}^{2}$ dark matter and a silicon crystal that promotes an electron to the conduction band (Fig. 3, left). Each Skipper CCD has a mass of only about 2 g. A first dark matter sensitivity test with a science-grade CCD has been conducted by the SENSEI Collaboration at Fermilab [22] (see Synopsis: Dark Matter Detector Proves its Sensitivity). The next step, planned for 2021, is to install a roughly 100 -g detector, consisting of about 50 Skipper CCDs, at Canada's underground laboratory SNOLAB. The DAMIC-M Collaboration is also planning to install a 1-kg detector at the Modane Underground Laboratory in France [23], and the US Department of Energy (DOE) is funding the R\&D of a multi-kg detector, called Oscura, which could take data later this decade [24]. The construction of these large-mass detectors is challenging. The CCDs need to be sufficiently low cost, the read-out electronics must read out many CCDs at once and with low noise, and the radioactive backgrounds must be controlled to incredible precision. 
Another device that has seen huge progress is the transition-edge sensor (TES), which has the potential to be a multipurpose dark matter detector for sensing electrons, photons, and phonons (see, for example, Refs. [25-27]). A TES consists of a superconducting film that's held very close to the film's superconducting transition temperature. When a tiny amount of energy is deposited into the film, the film switches to a normal state and its resistance jumps (Fig. 3, right). Recent results from the SuperCDMS Collaboration have showcased a large-area $\left(45 \mathrm{~cm}^{2}\right)$ phonon-seeking TES sensor with a baseline energy resolution of about $4 \mathrm{eV}$ [26]. Although smaller devices would have even better energy resolution, researchers are looking to improve the energy resolution further while keeping the detector size large. The DOE is funding the R\&D of TESSERACT, a project consisting of a liquid helium experiment as well as a gallium-arsenide and sapphire-based experiment, both with TES readout [24]. The initial goal is to have target masses of about $100 \mathrm{~g}$ to $1 \mathrm{~kg}$, and probe dark matter down to $\sim 1 \mathrm{MeV} / \mathrm{c}^{2}$. The ultimate goal of the TES technology is to sense single phonons, which would allow sensitivity to dark matter with masses as low as a few $\mathrm{keV} / \mathrm{c}^{2}$.

A third device that has seen rapid progress is a superconducting nanowire single-photon detector (SNSPD), which consists of a thin film of superconducting material across which a constant current is applied. After absorbing an infrared photon, a local hotspot is created, which locally breaks the superconductivity and creates a measurable voltage pulse. These devices are still small ( $m m$ scale) and new fabrication techniques and readout schemes are needed to increase their size. However, SNSPDs and other devices have the potential to probe dark matter well below the $\mathrm{GeV} / \mathrm{c}^{2}$ scale.

\section{The Background Challenge}

For all of these new detectors, physicists will have to find ways of increasing the sensitivity and the detector size while controlling potential dark counts and radiative backgrounds. From some of the first results that constrain dark matter down to the mass range of $1-100 \mathrm{MeV} / \mathrm{c}^{2}$, it's known that numerous low-energy events have been a problem (e.g., Refs. [3, 18-20, $22,23,25-28]$ ). Not surprisingly, the research on background sources has emphasized the energy range of WIMPs. But that focus is shifting as interest in lower-mass dark matter grows. For example, the first constraints on dark matter down to 5 $\mathrm{MeV} / \mathrm{c}^{2}$ were obtained with data taken by XENON10 in 2006 [3,
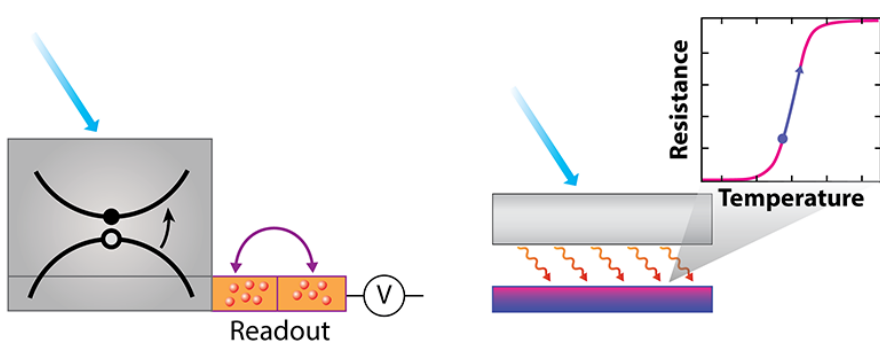

Figure 3: Two popular detectors for sub-GeV dark matter rely on solid-state effects to produce a signal. On the left, a silicon-based charge-coupled device produces an electrical current when a dark matter particle collides with the silicon and elevates an electron to the conduction band. On the right, a material impacted by a dark matter particle produces phonons or photons, which in turn induce a nearby superconducting film to switch to its normal state.

Credit: APS/Carin Cain

18]. Researchers are now making good progress in understanding and mitigating the large single- and few-electron backgrounds observed in these old data as well as in the data taken with more recent noble-liquid experiments $[19,20]$. Plans exist to use these new insights for controlling backgrounds to construct noble-liquid detectors of about $100 \mathrm{~kg}$ that will search for events containing one to a few electrons [24].

Other important background sources include radiative processes originating from high-energy backgrounds, which may be relevant for several sub-GeV detectors [29]. For example, one source of the problem is the nonconductive target or material surrounding the target. When electrons from radioactive decays or muons from cosmic rays interact with this material, they produce "Cherenkov" radiation. These interactions contribute to the single-electron events observed at SENSEI [22]. They are also plausibly behind a large fraction of the observed low-energy events at SuperCDMS-HVeV [26]. Fortunately, there are ways to mitigate this background. Steps include minimizing the amount of nonconductive material surrounding the detector, employing extensive passive and active shielding, and placing multiple detectors near each other to veto spurious events. As physicists continue exploring sub-GeV dark matter, they will likely identify other background sources that they will then characterize and mitigate. 


\section{How to Be Convinced of a Dark Matter Discovery}

After building the first dedicated low-threshold dark matter experiments, physicists will hopefully observe an unexplained excess. However, how can we as a physics community be convinced that dark matter has been discovered? The answer depends on the precise details of the excess, since not all statistically significant excesses are equally likely to be new physics. Here are five critical questions that need to be addressed: (1) Has the excess been observed by a second, independent collaboration? (2) Has the excess been observed with a second, independent technology? (3) Is it possible to construct a simple and viable dark matter model that explains the excess without being in conflict with other direct-detection, accelerator-based, astrophysical, or cosmological probes?

Occam's razor is not a scientific necessity, but physicists should be skeptical about models that add complexity for the sole reason of satisfying constraints from previous searches. (4) Are all the backgrounds understood and under control? (5) Are you sure about your answer to question (4)?

I'm confident that we will be able to answer these questions if and when we discover dark matter. However, these considerations argue strongly for supporting multiple small-scale approaches in the dark matter search, certainly at the R\&D stage. Also, even if a certain technology purports to cover a specific region in parameter space, we should support other independent approaches. Not only will they be needed to probe the full range of properties that dark matter may have, but they will also be needed to confirm a discovery. To this end, the fact that small-scale experiments have a relatively low cost makes them a worthwhile investment with a potentially enormous return.

Rouven Essig: C. N. Yang Institute for Theoretical Physics, Stony Brook University, Stony Brook, NY, USA

\section{REFERENCES}

1. M. Battaglieri et al., "US Cosmic visions: New ideas in dark matter 2017: Community report," arXiv:1707.04591.

2. R. Essig et al., "Direct detection of sub-GeV dark matter," Phys. Rev. D 85, 076007 (2012).

3. R. Essig et al., "First direct detection limits on sub-GeV dark matter from XENON10," Phys. Rev. Lett. 109, 021301 (2012).

4. P. W. Graham et al., "Semiconductor probes of light dark matter," Phys. Dark Universe 1, 32 (2012).
5. R. Essig et al., "Direct detection of sub-GeV dark matter with semiconductor targets," J. High Energy Phys. 2016, 46 (2016).

6. S. Derenzo et al., "Direct detection of sub-GeV dark matter with scintillating targets," Phys. Rev. D 96, 016026 (2017).

7. N. A. Kurinsky et al., "Diamond detectors for direct detection of sub-GeV dark matter," Phys. Rev. D 99, 123005 (2019).

8. S. M. Griffin et al., "SiC detectors for sub-GeV dark matter," arXiv:2008.08560.

9. C. Blanco et al., "Dark matter-electron scattering from aromatic organic targets," Phys. Rev. D 101, 056001 (2020).

10. Y. Hochberg et al., "Detecting superlight dark matter with Fermi-degenerate materials," J. High Energy Phys. 2016, 57 (2016); Y. Hochberg et al., "Detecting sub-GeV dark matter with superconducting nanowires," Phys. Rev. Lett. 123, 151802 (2019).

11. Y. Hochberg et al., "Directional detection of dark matter with two-dimensional targets," Phys. Lett. B 772, 239 (2017).

12. Y. Hochberg et al., "Detection of sub-MeV dark matter with three-dimensional Dirac materials," Phys. Rev. D 97, 015004 (2018).

13. C. Kouvaris and J. Pradler, "Probing sub-GeV dark matter with conventional detectors," Phys. Rev. Lett. 118, 031803 (2017).

14. M. Ibe et al., "Migdal effect in dark matter direct detection experiments," J. High Energy Phys. 2018, 194 (2018).

15. R. Essig et al., "Direct detection of nuclear scattering of sub-GeV dark matter using molecular excitations," Phys. Rev. Research 1, 033105 (2019); R. Budnik et al., "Direct detection of light dark matter and solar neutrinos via color center production in crystals," Phys. Lett. B 782, 242 (2018).

16. S. Knapen et al., "Detection of light dark matter with optical phonons in polar materials," Phys. Lett. B 785, 386 (2018).

17. S. A. Hertel et al., "Direct detection of sub-GeV dark matter using a superfluid ${ }^{4}$ He target," Phys. Rev. D 100, 092007 (2019).

18. J. Angle et al. (XENON10 Collaboration), "Search for light dark matter in XENON10 data," Phys. Rev. Lett. 107, 051301 (2011), [Erratum: Phys. Rev. Lett.110, 249901 (2013)].

19. P. Agnes et al. (The DarkSide Collaboration), "Constraints on sub-GeV dark-matter-electron scattering from the DarkSide-50 experiment," Phys. Rev. Lett. 121, 111303 (2018).

20. E. Aprile et al. (XENON Collaboration), "Light dark matter search with ionization signals in XENON1T," Phys. Rev. Lett. 123, 251801 (2019).

21. J. Tiffenberg et al. (SENSEI Collaboration), "Single-electron and single-photon sensitivity with a silicon Skipper CCD," 
Phys. Rev. Lett. 119, 131802 (2017).

22. L. Barak et al. (SENSEI Collaboration), "SENSEI:

Direct-detection results on sub-GeV dark matter from a new Skipper CCD," Phys. Rev. Lett. 125, 171802 (2020).

23. N. Castelló-Mor (DAMIC-M Collaboration ), "DAMIC-M experiment: Thick, silicon CCDs to search for light dark matter," Nucl. Instrum. Meth. A 958, 162933 (2020).

24. Basic Research Needs for Dark-Matter Small Projects New Initiatives, U.S. Department of Energy, 2019.

25. A. H. Abdelhameed et al. (CRESST Collaboration), "First results from the CRESST-III low-mass dark matter program," Phys. Rev. D 100, 102002 (2019).

26. D. Amaral et al. (SuperCDMS Collaboration), "Constraints on low-mass, relic dark matter candidates from a surface-operated SuperCDMS single-charge sensitive detector," arXiv:2005.14067.

27. I. Alkhatib et al. (SuperCDMS Collaboration), "Light dark matter search with a high-resolution athermal phonon detector operated above ground," arXiv:2007.14289.

28. Q. Arnaud et al. (EDELWEISS Collaboration), "First germanium-based constraints on sub-MeV dark matter with the EDELWEISS experiment," Phys. Rev. Lett. 125, 141301 (2020).

29. P. Du et al., "Sources of low-energy events in low-threshold dark matter detectors," arXiv:2011.13939. 